\title{
Blood-brain barrier invasion by group B Streptococcus depends upon proper cell-surface anchoring of lipoteichoic acid
}

\author{
Kelly S. Doran, ${ }^{1}$ Erin J. Engelson, ${ }^{1}$ Arya Khosravi, ${ }^{1}$ Heather C. Maisey, ${ }^{1}$ Iris Fedtke, ${ }^{2}$ Ozlem Equils, ${ }^{3}$ \\ Kathrin S. Michelsen,, ${ }^{3}$ Moshe Arditi, ${ }^{3}$ Andreas Peschel, ${ }^{2}$ and Victor Nizet ${ }^{1}$ \\ 'Department of Pediatrics, Division of Infectious Diseases, UCSD School of Medicine, La Jolla, California, USA. ${ }^{2}$ Universität Tübingen, \\ Tübingen, Germany. ${ }^{3}$ Cedars-Sinai Medical Center, Los Angeles, California, USA.
}

\begin{abstract}
Group B streptococci (GBSs) are the leading cause of neonatal meningitis. GBSs enter the CNS by penetrating the blood-brain barrier (BBB), which consists of specialized human brain microvascular endothelial cells (hBMECs). To identify GBS factors required for BBB penetration, we generated random mutant libraries of a virulent strain and screened for loss of hBMEC invasion in vitro. Two independent hypo-invasive mutants possessed disruptions in the same gene, invasion associated gene (iagA), which encodes a glycosyltransferase homolog. Allelic replacement of iagA in the GBS chromosome produced a 4-fold decrease in hBMEC invasiveness. Mice challenged with the GBS $\triangle$ iagA mutant developed bacteremia comparably to WT mice, yet mortality was significantly lower $(20 \%$ vs. $90 \%$ ), as was the incidence of meningitis. The glycolipid diglucosyldiacylglycerol, a cell membrane anchor for lipoteichoic acid (LTA) and predicted product of the IagA glycosyltransferase, was absent in the $\triangle i a g A$ mutant, which consequently shed LTA into the media. Attenuation of virulence of the $\triangle i a g A$ mutant was found to be independent of TLR2-mediated signaling, but bacterial supernatants from the $\triangle$ iagA mutant containing released LTA inhibited hBMEC invasion by WT GBS. Our data suggest that LTA expression on the GBS surface plays a role in bacterial interaction with $\mathrm{BBB}$ endothelium and the pathogenesis of neonatal meningitis.
\end{abstract}

\section{Introduction}

Bacterial meningitis is the most common serious infection of the CNS and a major cause of death and disability in children worldwide (1). Even with proper treatment, 3-25\% of affected children may die or suffer permanent disability with sequelae that include cognitive deficits, cerebral palsy, blindness, deafness, or seizures. In order to produce meningitis, blood-borne bacteria must interact with and breech the blood-brain barrier (BBB). This barrier, responsible for maintaining biochemical homeostasis within the CNS, consists principally of a single layer of specialized brain microvascular endothelial cells (BMECs) exhibiting continuous tight junctions and lacking pinocytosis (2). Penetration of the BBB by a bacterial pathogen reflects a complex interplay between the host endothelium and microbial surface components.

The Gram-positive bacterium Streptococcus agalactiae, known as group B Streptococcus (GBS), is the leading cause of meningitis in newborn infants. GBS strains of all 9 described capsular serotypes can produce meningitis; however, serotype III strains account for a disproportionate share ( $\sim 80-85 \%)$ of CNS isolates (3). In animal studies, the risk of GBS meningitis has been correlated with the magnitude and duration of bacteremia, and a perivascular distribution of bacteria within the CNS reflects a tropism of the organism for BBB endothelium (4). To probe this interaction, one can take advantage

Nonstandard abbreviations used: BBB, blood-brain barrier; BLAST, Basic Local Alignment Search Tool; Cm, chloramphenicol; CRAMP, cathelin-related antimicrobial peptide; DGlcDAG, [Glucose $(\beta 1-6)$ Glucose $(\beta 1-3)$ (gentiobiosyl)diacylglycerol]; Em, erythromycin; GBS, group B Streptococcus; GP, glycerol phosphate; hBMEC, human brain microvascular endothelial cell; iagA, invasion-associated gene; LTA, lipoteichoic acid; MGlcDAG, monoglycosyldiacylglycerol; ORF, open reading frame. Conflict of interest: The authors have declared that no conflict of interest exists.

Citation for this article: J. Clin. Invest. 115:2499-2507 (2005).

doi:10.1172/JCI23829. of a tissue culture model of the human BBB consisting of immortalized human brain microvascular endothelial cells (hBMECs) that maintain the morphologic and functional characteristics of primary brain endothelium (5). In previous studies, we found that GBS efficiently invaded hBMECs and survived intracellularly (6). Electron microscopy revealed that GBS exploited host internalization pathways, adhering tightly to the hBMEC surface, inducing microfilament- and microtubule-dependent cytoskeletal rearrangement, and entering the cell within a membrane-bound endocytotic vacuole (6). GBS invasion of hBMECs required active bacterial DNA, RNA, and protein synthesis, which points to the involvement of specific geneencoded bacterial virulence determinants.

We hypothesized that GBS invasion of hBMECs is a primary and essential step in the pathogenesis of neonatal meningitis. The specific molecular factors that allow GBS to invade hBMECs, however, are unknown. In the present manuscript, we applied genetic techniques of random transposon and plasmid integrational mutagenesis to discover a GBS gene essential for efficient hBMEC invasion in vitro. We then assessed the impact of this mutation on GBS BBB penetration and virulence in vivo. As a result, a novel role of cell membrane-anchored lipoteichoic acid (LTA) in GBS disease pathogenesis was established.

\section{Results}

Identification of a GBS gene associated with hBMEC invasion. To identify novel GBS factors involved in hBMEC invasion, we constructed a random Tn917 mutagenesis library of virulent serotype III GBS strain $\mathrm{COH} 1$ and a random plasmid integrational mutagenesis library of the isogenic noncapsular strain COH1-13. The complementary mutagenesis strategies were employed to enhance saturation of the bacterial genome in the face of potential confounding factors such as "hot spots" for transposon insertion. Approximate- 

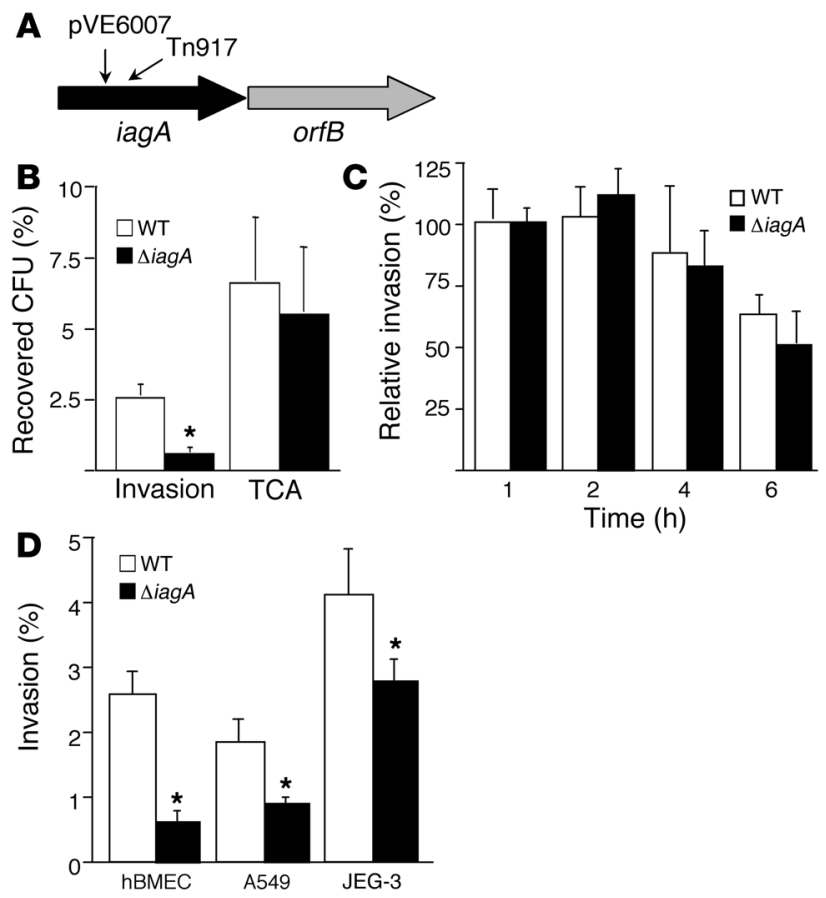

ly 5,000 Tn917 mutants and 1,500 plasmid integrational mutants were screened in a microtiter plate assay for hBMEC invasion, which yielded 20 and 7 candidates, respectively, with invasion levels of less than $50 \%$ of those of the parent strain on repeated measurements. Chromosomal sequence analysis of candidates revealed 2 independent mutants (COH1:IM8 with Tn917 and COH1-13: $\mathrm{H} 3$ with plasmid integration) with disruptions in the same open reading frame (ORF) of $999 \mathrm{bp}$, which we have designated invasionassociated gene (iagA) (Figure 1A). Chromosome walking revealed a second ORF (orfB) of 1,334 bp immediately downstream (2 bp) of iagA. Basic Local Alignment Search Tool (BLAST) analysis identified the presence of both ORFs in the 2 published GBS genomes: the type III strain NEM316 (7), where they are designated gbs0682 and gbs0683; and type V strain $2603 \mathrm{~V} / \mathrm{R}(8)$, where they are designated sag0709 and sag0710. A predicted $\alpha$ amylase family protein precedes the iagA gene with an intervening region of $45 \mathrm{bp}$, and a threonyl-transfer RNA synthetase is $446 \mathrm{bp}$ downstream of orf $B$. The predicted protein products of $i a g A$ and $\operatorname{orf} B$ share homology with putative sugar transferases from Gram-positive bacteria, including the human pathogens Streptococcus pyogenes (68\% identity and $80 \%$ similarity), Enterococcus faecalis (53\% identity and $71 \%$ similarity), and Streptococcus mutans (69\% identity and $83 \%$ similarity). In almost all cases, the 2 ORFs are present in the same arrangement. The predicted iag $A$ protein shared lesser but still significant degrees of homology with proteins with characterized function, including a 1,2-diacylglycerol-3-glucose (1-2)-glucosyltransferase from Acholeplasma laidlawii (45\% identity and 62\% similarity) (9) and the $\beta$-lactam resistance factor $c p o A$ from Streptococcus pneumoniae (24\% identity and 44\% similarity) (10). Using internal PCR primers, we found the iagA gene to be present in GBS isolates representing serotypes Ia, III, V, and VI (data not shown).

Confirmation of the role of iagA in hBMEC invasion by targeted mutagenesis. The identification of mutated iagA gene sequence in 2 independent mutants with decreased hBMEC invasiveness strongly suggested a role for this chromosomal locus in the invasion phe-

\section{Figure 1}

The newly identified $\triangle$ iagA gene contributes to host cell invasion. (A) Schematic representation of the GBS iagA region and the position of the Tn917 or pVE6007 insertion in original mutants, indicated by small arrows. (B and C) Interaction of WT and $\triangle i a g A$ with hBMECs. (B) Invasion and total cell-associated (TCA; surface-adherent plus intracellular) bacteria. (C) Intracellular survival over time. (D) Invasion of cell lines hBMEC, A549 (lung epithelial cells), and JEG-3 (chorion epithelial cells) by WT and $\triangle$ iagA strains. ${ }^{*} P<0.005$.

notype. To assess whether this association was specifically attributable to the iagA gene, we performed precise, in-frame allelic replacement of $i g g A$ in WT GBS COH1 to generate the isogenic mutant COH1 $\operatorname{ciagA}$. We then performed quantitative hBMEC invasion assays as described previously (6) in an in vitro BBB model. An inoculum of $10^{5} \mathrm{CFU} /$ well of COH1 1 iagA resulted in an MOI of 1-3; data are expressed as percent intracellular GBS relative to hMBECs after 2 hours incubation. As shown in Figure 1B, COH1 $\triangle$ iagA exhibited a 4-fold decrease in hBMEC invasion compared with the WT parent strain $(P<0.0001)$. We also tested GBS invasion of a murine BMEC cell line (pBEND; ATTC) and found that the $\triangle i a g A$ mutant exhibited a similar hypo-invasive phenotype relative to the WT strain $(P<0.0002$; data not shown). Additionally, because certain bacteria exhibit increased invasion on activated eukaryotic cells, we performed invasion assays in which the hBMEC monolayers were pretreated with the proinflammatory cytokines TNF- $\alpha$, IL- 6 , or IL-8. We found that pretreatment with the various cytokines did not reverse the observed hypo-invasive phenotype of the $\triangle i a g A$ mutant (data not shown).

The iagA gene does not affect either hBMEC adherence or intracellular survival. Two additional phenotypic properties that could lead to changes in the calculated hBMEC intracellular invasion in our assay would be (a) the affinity of GBS adherence to the hBMEC surface and (b) the ability of GBS to survive intracellularly after endocytotic uptake. We thus performed additional experiments to assess the level of total cell-associated (surface-adherent plus intracellular) bacteria (Figure 1B). In these assays, GBSs were quantified from hBMEC monolayers after vigorous washing but prior to the addition of extracellular antibiotics (see Methods). Consistent with previously reported results (6), approximately $38 \%$ of total hBMEC-associated WT strain COH1 had invaded the intracellular compartment. Upon administration of $\mathrm{COH} 1 \Delta i a g A$, there was a slight decrease in the total number of cell-associated bacteria; however, this decrease was not statistically significant, and, furthermore, only $9 \%$ of total hBMEC-associated mutant GBSs were present intracellularly. To assess whether the $\triangle i a g A$ mutant might be impaired in survival after hBMEC entry, we performed a modified invasion assay in which the number of intracellular GBSs was quantified at different time points after addition of antibiotics to kill extracellular bacteria. As shown in Figure 1C, COH1 and $\mathrm{COH} 1 \Delta i a g A$ exhibited a similar decrease in intracellular survival over time. The sum of our results indicate the iagA gene plays a specific role in facilitating GBS hBMEC invasion, without significantly altering hBMEC adherence or intracellular survival.

Effect of iagA mutation on invasion of other epithelial cell barriers. Invasion of chorion epithelium (11) and lung epithelium (12) are believed to play a role in earlier stages of GBS neonatal disease pathogenesis (13). To examine whether the effect of this iagA mutation on GBS invasion is restricted to BBB endothelium, we performed invasion assays using (a) human lung epithelial cell line A549 and (b) human 
A

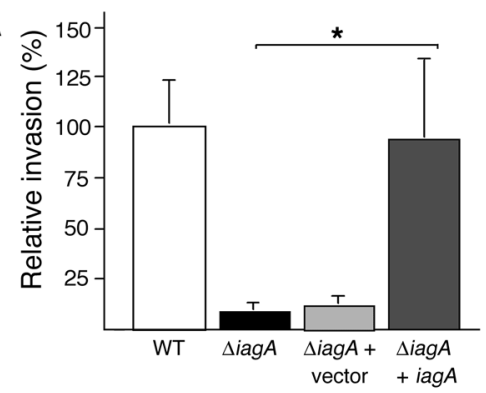

B

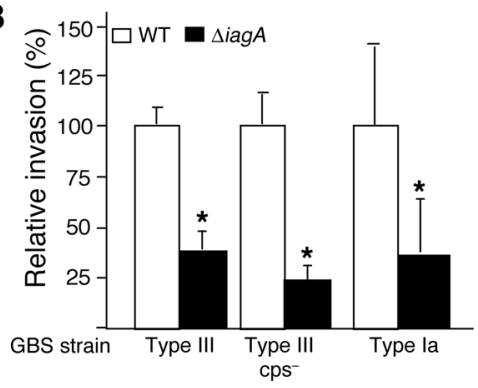

Figure 2

Analysis of the hBMEC-invading $\triangle i a g A$ mutant phenotype. (A) Expression of iagA gene restores hBMEC invasion. (B) Invasion of $\triangle i a g A$ mutants in GBS type III capsule-deficient $\left(\mathrm{cps}^{-}\right)$strains. Percent invasion is normalized to that of the WT strain. ${ }^{*} P<0.005$.

chorion epithelial cell line JEG-3. We found that $\triangle i a g A$ invasion was significantly attenuated in both epithelial cell lines, though not quite to the extent that it was in hBMECs (Figure 1D). We conclude that iagA may contribute to GBS invasive potential in several cell types relevant to different stages of disease pathogenesis.

Exclusion of additional pleiotropic effects relevant to hBMEC invasion assays. WT strain $\mathrm{COH} 1$ and mutant $\mathrm{COH} 1 \Delta i a g A$ demonstrated equivalent growth kinetics in Todd-Hewitt broth (THB) and the RPMI-based culture medium used in our in vitro hBMEC assays, as well as similar gross morphology and chain length (data not shown). The surface-associated GBS $\beta$-hemolysin/cytolysin $(\beta \mathrm{h} / \mathrm{c})$ plays a role in hBMEC injury (6) and inflammatory activation (14) and could affect interpretation of tissue culture studies; however, no differences in $\beta \mathrm{h} / \mathrm{c}$ activity were observed between the WT and $\triangle i a g A$ mutant strains (data not shown). The sialylated GBS exopolysaccharide capsule appears to mitigate hBMEC invasion (6) and inflammatory activation (14). However, our analysis of acidreleased GBS sialic acids by 1,2-diamino-4,5-methylenedioxybenzene (DMB) derivatization and reverse-phase HPLC (15) revealed no difference in capsular sialic acid content between WT and $\Delta i a g A$ mutant strains (data not shown). The hypo-invasive phenotype of a targeted deletion of the iagA gene in a capsule-negative GBS strain (Figure 2B) further supports a role for iagA in hBMEC invasion that is independent of capsule. Finally, GBS iagA shares some homology with the cpoA gene of $S$. pneumoniae. Mutations in the сроA gene conferred piperacillin resistance, decreased competence for transformation, and a decrease in penicillin-binding protein la expression (10). We found, however, that the minimum inhibitory concentrations of COH1 and COH1 $\operatorname{Cig} A$ to penicillin $(0.019 \mu \mathrm{g} /$ $\mathrm{ml})$ and gentamicin $(6.25 \mu \mathrm{g} / \mathrm{ml})$ were identical. Moreover, omission of penicillin from the hBMEC invasion assay did not affect the relative decrease in hBMEC invasion by the $\triangle i a g A$ mutant compared with the WT parent strain.
Single-gene complementation of the iagA mutant restores bBMEC invasion. To establish unambiguously that the GBS iagA gene specifically contributes to the hBMEC invasion phenotype, we performed single-gene complementation analysis. The iagA gene was amplified from WT strain COH1, cloned in expression vector $\mathrm{pDCerm}$ to yield pDCiagA, and used to transform the COH1 1 iagA mutant. As a vector-only control, COH1 1 iagA was also transformed with pDCerm. When tested in the hBMEC invasion assay, complementation of the $\triangle i a g A$ mutant with pDCiagA restored invasion to WT levels, while the $\triangle i a g A$ mutant transformed with pDCerm alone retained its hypo-invasive phenotype (Figure 2A). These studies corroborate a specific requirement for iag $A$ in hBMEC invasion and rule out the possibility that secondary chromosomal mutations or unanticipated polar effects on adjacent genes contribute to the hypo-invasive phenotype of the COH1 $\mathrm{CiagA}$ allelic exchange mutant.

Effect of the iagA mutation in other GBS strains. Serotype III, the most common GBS serotype associated with meningitis, accounts for almost half of GBS meningitis isolates in the United States based on surveillance of isolates conducted by the Active Bacterial Core in 1998-2003 (S. Schrag, personal communication); the next most common GBS meningitis serotypes are types Ia and V (S. Schrag, personal communication). According to recently published data from the United Kingdom, roughly 35\% of total infections with serotype III were complicated by meningitis, compared with approximately $15 \%$ of infections with serotype Ia or V strains (16). To determine whether the effect of iagA LTA anchoring on GBS hBMEC invasion was restricted to serotype III, we generated a plasmid integrational mutant of the iagA gene in GBS strain A909, a serotype Ia isolate from the CSF of a neonate with meningitis. We found that disruption of iagA in the serotype Ia background is also associated with a significant decrease in hBMEC invasion (Figure 2B). Allelic replacement of the $i a g A$ gene in a serotype III GBS mutant lacking capsular polysaccharide also reduced invasion, which indicated that the type III capsule per se is not required for surface-expressed LTA to promote hBMEC uptake. Because GBS isolates of multiple serotypes are capable of hBMEC invasion (6), we conclude that LTA anchoring is likely of general importance as a virulence phenotype of the pathogen.

Role of iagA in bloodstream survival and penetration of the $B B B$ in a murine model of meningitis. Our results thus far suggest a primary role for the iagA gene in GBS invasion and penetration of the BBB. We hypothesized that this in vitro phenotype would translate into a diminished ability to penetrate the $\mathrm{BBB}$ and produce meningitis in vivo. Using a murine model of hematogenous meningitis (14), we infected groups of mice $(n=10)$ i.v. with the WT GBS strain $\mathrm{COH} 1$ or the isogenic COH1 $\operatorname{liagA}$ mutant. Twenty-four hours after challenge, the levels of GBS detected in the blood of each group were essentially identical (Figure 3A). These results are consistent with those of additional studies that demonstrated no significant difference between the ability of the WT and $\Delta i a g A$ strains to survive in whole human blood (data not shown). Additionally, because antimicrobial peptides are expressed in innate host immune cells, such as neutrophils, and have been shown to provide a first line of defense against invasive streptococcal infections (17), we compared the sensitivity of the WT and $\triangle i a g A$ strains to the mouse cathelin-related antimicrobial peptide (CRAMP). Analysis of bacterial killing kinetics revealed no difference in sensitivity of the WT and $\triangle i a g A$ strains to CRAMP-mediated killing (Figure 3B), which further suggests that the $\triangle i a g A$ mutant is not generally compromised in bloodstream survival. Despite their initial similarities 

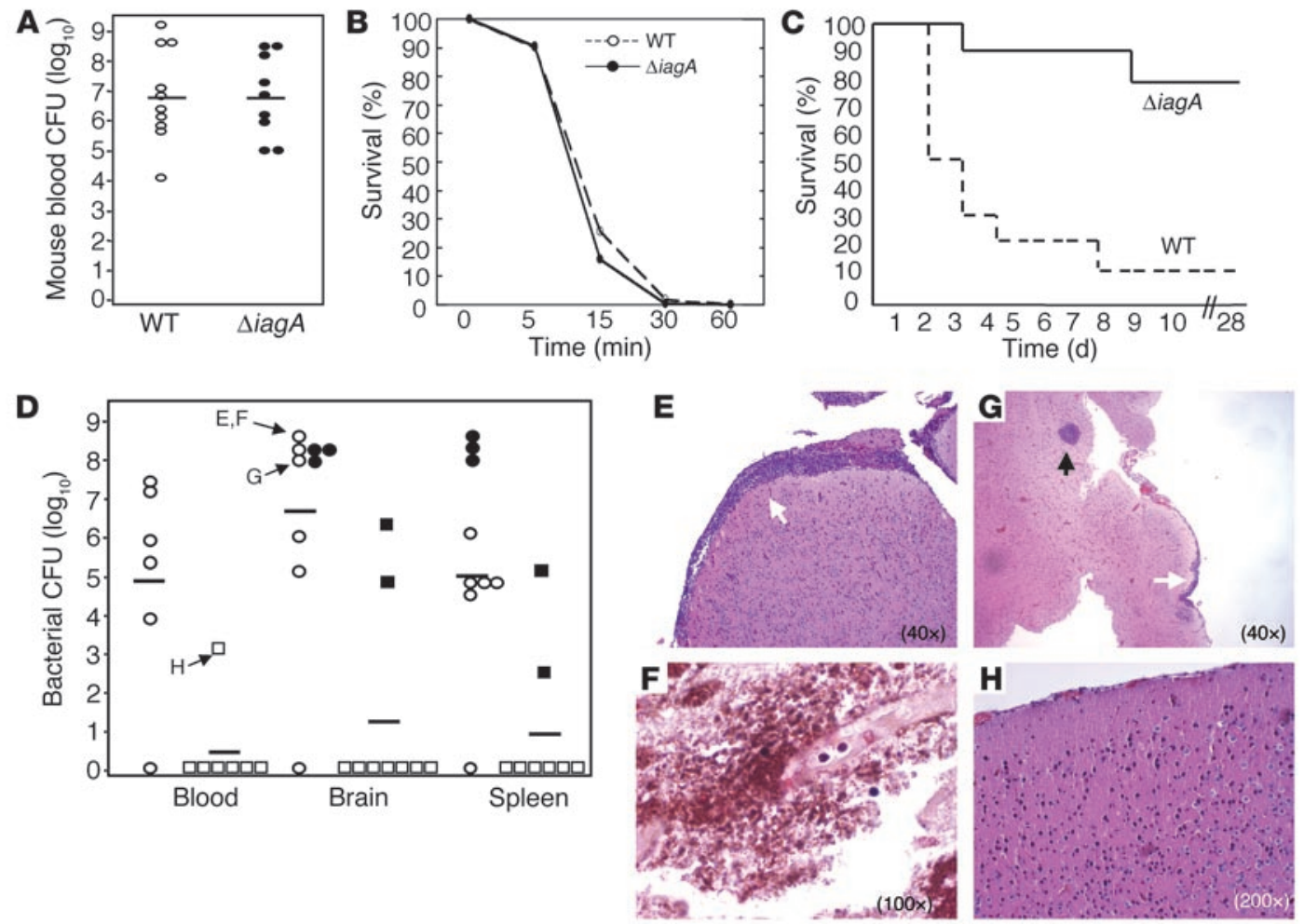

Figure 3

In vivo analysis of the $\triangle i a g A$ mutant using a mouse model. Deletion of iagA does not effect GBS survival in mouse blood (A) or sensitivity to antimicrobial peptide CRAMP (B) but does render GBS less pathogenic in vivo. Groups of 10 mice were inoculated i.v. with WT or $\triangle i a g A$ GBS strains. (C) Kaplan-Meier survival plot. (D) Bacterial counts (CFU) in mouse blood, brain, and spleen at time of death of WT (circles) and $\Delta i a g A$ (squares); filled symbols represent mice that died before the experiemental endpoint. Histopathology of H\&E- $(\mathbf{E}-\mathbf{H})$ or $\mathbf{G r a m}-\mathrm{stained}(\mathbf{F})$ brain tissues of individual mice (identified by arrows in D). (E and G) Samples from mice infected with WT GBS show meningeal thickening and cellular infiltration (open arrows) and bacterial microabscess formation (filled arrow). (F) Gram stain of mouse infected with WT GBS. (H) A sample from the mouse infected with the iagA mutant shows normal brain histopathology.

in establishing a high-grade bacteremia in the mouse and similar sensitivities to CRAMP, the WT GBS strain went on to cause significantly higher mortality than the isogenic $\Delta i a g A$ mutant (Figure $3 \mathrm{C})$. By the second day, half of the mice infected with $\mathrm{COH} 1 \mathrm{had}$ died, and all but 1 died by the seventh day. In contrast, only 2 mice infected with the $\Delta i a g A$ mutant died, while the rest of the mice were able to clear the infection and survive to the experimental endpoint of 28 days. At the time of death (or upon euthanasia at day 28), blood, spleen, and brain were harvested from each mouse for quantitative bacterial culture. As shown in Figure 3D, the WT strain exhibited significantly higher final bacterial loads and penetrated the BBB more frequently than the isogenic $\triangle i a g A$ mutant. Mean counts for bacteria isolated from the brains of mice infected with COH1 $\triangle$ iagA were 3,500-fold lower than those calculated for mice infected with WT strain COH1 $(P<0.0002)$. These data demonstrate that the $\operatorname{igg} A$ gene contributes significantly to the ability of GBS to penetrate the BBB in vivo. Representative histopathology of brains from infected mice is shown in Figure 3, E-H. Mice that succumbed to infection had clear evidence of GBS meningitis with associated neutrophilic inflammatory responses, with the degree of histopathologic abnormality correlating closely with the magnitude of bacterial counts in the brain tissue.

Glycolipid analysis and effect on LTA. The predicted product of the iagA gene shares homology with glycosyltransferases, specifically the well-studied 1,2-diacylglycerol-3-glucose (1-2)-glucosyltrans- ferase from A. laidlawii (9) that has been shown to synthesize the cell wall-associated [Glucose $(\beta 1-6)$ Glucose $(\beta 1-3)$ (gentiobiosyl)d iacylglycerol] (DGlcDAG) from the precursor monoglycosyldiacylglycerol (MGlcDAG). Therefore, we analyzed the glycolipid content of the $\Delta$ iagA mutant compared with that of the WT parental strain by thin-layer chromatography. As shown in Figure 4A, WT GBS COH1 made both glycoplipids (MGlcDAG and DGlcDAG), while the iagA mutant failed to synthesize DGlcDAG. A strain of Staphylococcus aureus, SA113 (18), that contains only DGlcDAG in detectable amounts is shown as control. This experiment confirmed the predicted activity of the GBS $i a g A$ gene product as a DGlcDAG synthase. Because DGlcDAG is known to act as the glycolipid anchor for LTA in several Gram-positive bacteria (19), we examined whether LTA anchoring was impaired in the GBS $\Delta i a g A$ mutant. Figure 4, B-D, shows the results of ELISA performed using an mAb against LTA (20) on whole cells and supernatants from WT GBS COH1 and mutant COH1 1 iagA. A marked increase in LTA released into the supernatant from the $\triangle i a g A$ mutant compared with the WT was found at both the logarithmic and stationary phases of growth $(P<0.00003$; Figure 4B). We also detected more LTA associated with the surface of the COH1 1 iagA mutant compared with the WT (Figure 4C). In contrast, when the iagA gene was disrupted in a capsule-negative GBS strain, COH1-13: $\mathrm{H} 3$, the mutation produced a decrease in measured LTA on the bacterial surface (Figure 4D). From these results, we conclude that 

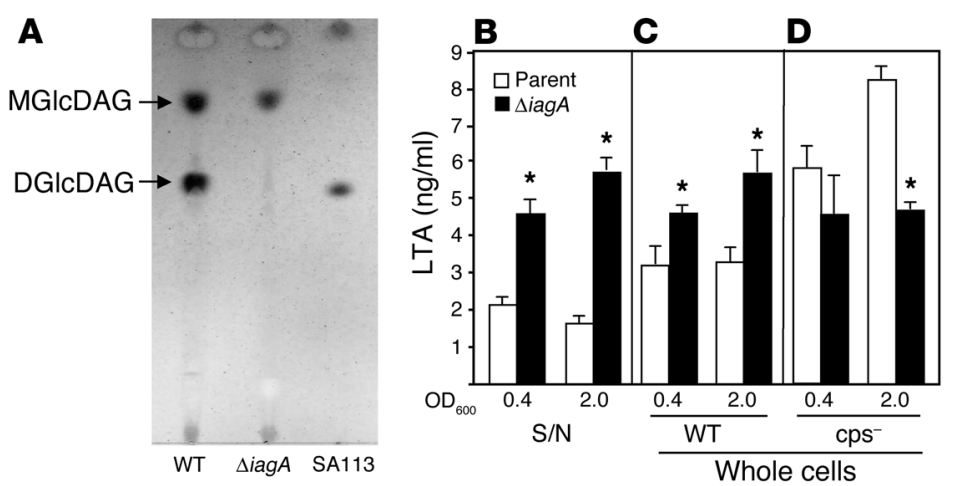

\section{Figure 4}

Glycolipid and LTA analysis. (A) Comparison of glycolipid content of WT and $\triangle$ iagA GBS strains using thin-layer chromatography. SA113 is a WT S. aureus control. ELISA analysis of LTA of supernatant (S/N) (B) or whole cells (C) from WT and $\Delta i a g A$ strains or whole cells in a capsule-negative genetic background (D). Supernatants or cells were analyzed at logarithmic $\left(\mathrm{OD}_{600}=0.4\right)$ or stationary $\left(\mathrm{OD}_{600}=2.0\right)$ phase as indicated. ${ }^{*} P<0.01$. when the unanchored LTA is released from the cell wall by the GBS $\triangle i a g A$ mutant, some of it becomes trapped in the thick polysaccharide capsule and remains detectable in our ELISA of whole cells.

Role of TLR2 on LTA-mediated penetration of the BBB. LTA can serve as a pattern-recognition molecule for TLR-mediated activation of the host innate immune response to Gram-positive pathogens (21, 22). We investigated whether differences in LTA signaling via TLR2 between $\triangle i a g A$ mutant and WT could contribute to the observed hypo-invasive phenotype and diminished virulence. We first compared WT GBS and the $\triangle i a g A$ mutant for mortality and BBB invasion in WT C57BL/6 and TLR2-/- mice (Figure 5, A and B). We found that TLR2 ${ }^{-1-}$ mice had greatly enhanced susceptibility to WT GBS infection, as all knockout mice challenged with the WT bacterium died within 24 hours. In contrast, only $1 / 5$ of the TLR2-/- mice challenged with the $\triangle i a g A$ mutant died by our 48 -hour endpoint. WT and $\triangle i a g A$ mutant bacterial counts in the blood were similiar at 6 hours in both WT or TLR2 $/$ - mice; however, the $\triangle$ iagA mutant failed to penetrate the BBB in 4/5 WT mice and $2 / 5$ TLR2 ${ }^{-1-}$ mice. Among mice challenged with WT GBS, bacteria were cultured in the brain of 4/5 WT mice, and evidence of bacterial CNS invasion was observed grossly in all TLR2 ${ }^{-/-}$mice that succumbed within the first day of infection. Of note, enhanced virulence of WT GBS in TLR2 $/-$ mice was recently reported by another group (23).

We next asked whether the iagA mutation affects activation of inflammatory pathways intrinsic to hBMECs. The hBMEC line has been shown to express TLR2 by RT-PCR analysis (24). In our earlier microarray analysis of hBMECs (14), we found that the gene for the chemokine IL-8, a common target of TLR/NF- $\mathrm{KB}-$ mediated activation, was the single most strongly induced gene following GBS stimulation (> 60-fold upregulation), a finding confirmed by ELISA for IL-8 protein. We stimulated hBMECs with WT GBS and the isogenic $\triangle i a g A$ and found no differences in IL-8 secretion at 4 and 8 hours (Figure 5C). We further analyzed the question of potential differences in TLR2 signaling by expressing TLR2 (and, as control, TLR4) in a human endothelial cell line containing an NF-кB luciferase reporter construct. GBS activated NF- $\mathrm{BB}$ in a TLR2-specific fashion, but no significant differences between the WT and $\Delta i a g A$ mutant strains were identified (Figure 5D). Combining these findings with our in vivo results, we concluded that the attenuation of virulence of the $\triangle i$ igg mutant is likely to be independent of TLR2.

Released LTA inhibits GBS invasion. As surface-anchored LTA appeared to facilitate GBS hBMEC entry, we hypothesized that released LTA could competitively inhibit the cellular invasion process. To examine this possibility, we performed a standard GBS hBMEC invasion assay in the presence of added culture supernatant collected from the $\triangle i a g A$ mutant or, as a control, the WT strain. Whereas WT supernatant stimulated enhanced GBS hBMEC invasion, a phenomenon we have observed before (K. Doran, unpublished observation), the supernatant from the $\triangle i a g A$ mutant had exactly the opposite effect, leading to significantly decreased GBS invasion $(P<0.007$; Figure 6).

\section{Discussion}

Our targeted allelic replacement and complementation studies reveal a unique requirement for the novel gene iagA in $\mathrm{BBB}$ penetration by GBS, the leading agent of neonatal bacterial meningitis. Decreased invasion of hBMECs by the GBS $\triangle i a g A$ mutant in vitro was correlated with a reduced risk for development of meningitis and markedly diminished lethality in vivo. These results corroborate the usefulness of the in vitro BBB model in the study of hostpathogen interactions and discovery of candidate virulence factors in other meningeal pathogens such as Streptococcus pneumoniae and E. coli (25-27). Deletion of iagA did not affect other key steps in the pathogenesis of GBS meningitis, including bloodstream survival, hBMEC adherence, and intracellular survival. Thus the iagAencoded phenotype of GBS has a specific function in promoting hBMEC uptake of the pathogen.

BLAST analysis of the GBS iagA gene and its predicted protein product revealed a conserved domain motif of the group 1 glycosyltransferases. Glycosyltransferases are carbohydrate-interacting enzymes involved in the synthesis of complex carbohydrate structures, and members of this family transfer activated sugars to a variety of substrates, including glycogen, fructose-6-phosphate, and lipopolysaccharides. In bacterial systems, glycosyltransferases play defined roles in biosynthesis of integral cell wall polysaccharides, lipopolysaccharides, and exopolysaccharide capsules. For example, the cpsE gene of serotype III GBS encodes a glycosyltransferase that initiates synthesis of the capsular polysaccharide repeat unit, such that $\Delta c p s \mathrm{E}$ mutants fail to produce a surface capsule $(28,29)$. Virulence of nonencapsulated mutants of serotype III GBS is greatly attenuated as a result of increased susceptibility to complement-mediated opsonophagocytic killing (30, 31). However, the same nonencapsulated mutants show increased invasion of hBMECs in vitro (6), which suggests that removal of the capsule may increase access to other GBS surface components capable of triggering the $\mathrm{BBB}$ endothelium to internalize the bacterium. In the present study, no changes in total encapsulation or capsular sialic acid content were detected in the GBS $\Delta i a g A$ mutant, and elimination of iagA resulted in decreased hBMEC invasion in both WT and acapsular $(\Delta c s p \mathrm{E})$ strains. These data suggested that the 

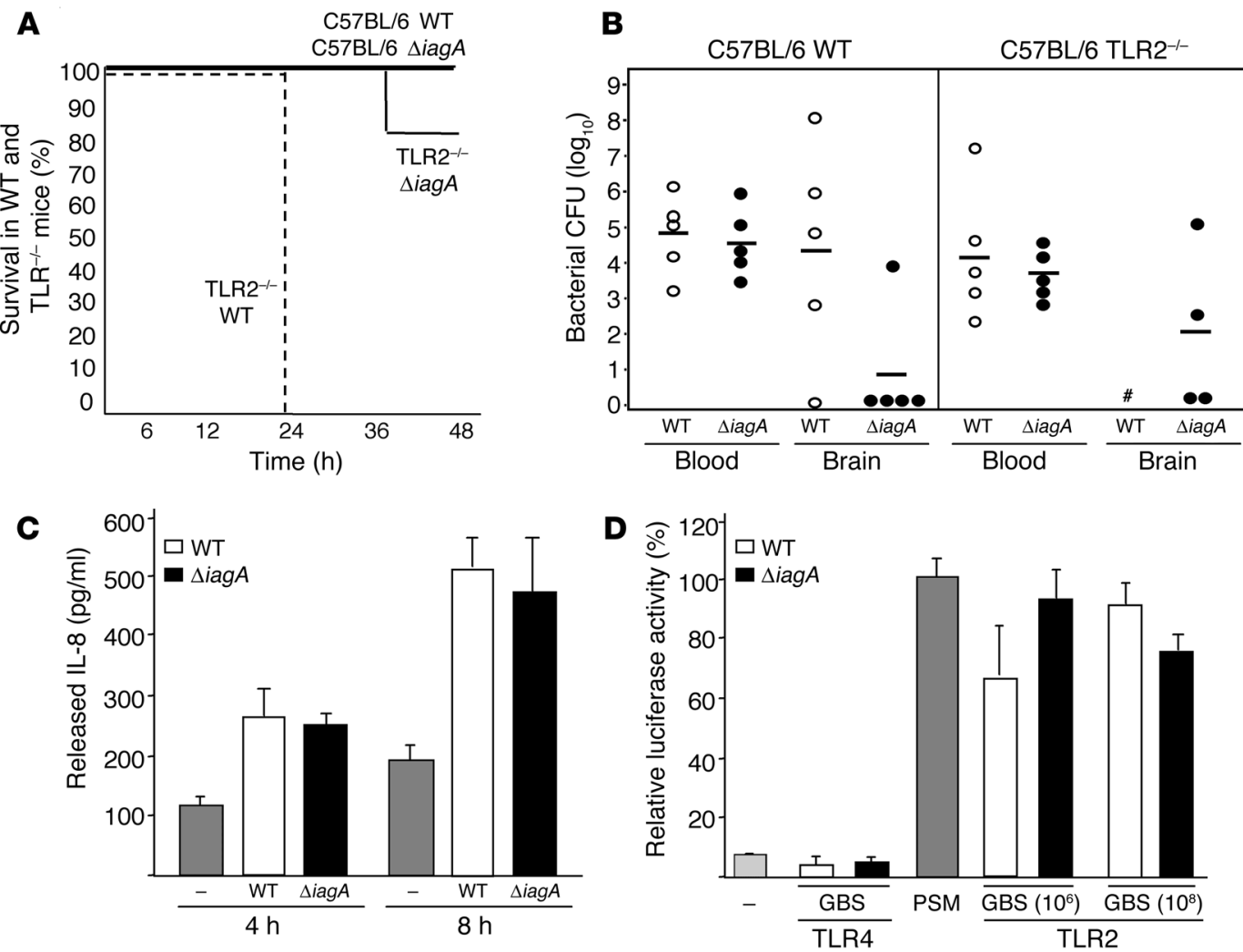

Figure 5

Absence of TLR2 does not effect attenuation of the $\triangle$ iagA mutant. (A) Kaplan-Meier survival plot of WT C57BL/6 and TLR2 ${ }^{-/-}$mice infected with WT or $\triangle$ iagA mutant GBS. (B) Bacterial counts (CFU) in mouse blood (at 6 hours) and in brain and at time of death (48 hours). "Bacterial brain counts were not determined because all TLR2 ${ }^{-1-}$ mice infected with WT GBS died before the experimental endpoint of 48 hours. (C) Detection of IL-8 released by hBMECs after stimulation with WT or $\triangle$ iagA mutant GBS using ELISA. (D) HMECs that expressed TLR2 or TLR4 were transfected with an NF-kB-luciferase reporter. Subsequently, cells were stimulated with 2 doses of GBS, and luciferase activity was determined by luminometry.

phenotype encoded by $\operatorname{iag} A$ was a surface factor that could mediate GBS-hBMEC interactions in a capsule-independent fashion.

The amphiphilic molecule LTA is a component of most Grampositive cell walls and is known to participate in host cell attachment by certain pathogens including S. pyogenes (32). A glycerol phosphate (GP) polymer that extends through the peptidoglycan layer of the cell wall, LTA is attached to the cytoplasmic membrane via the hydrophobic interactions of its glycolipid anchor (33). The GBS LTA contains complex lipids and short-chain fatty acids and averages 22 GP units in length; very little of the polymer is released from the cell under normal growth conditions $(34,35)$. In a number of streptococci, staphylococci, and bacilli studied, LTA is anchored to the cell membrane via DGlcDAG (19). We found homology of the predicted GBS iagA gene product with a glycosyltransferase of the cell wall-deficient bacterium $A$. laidlawii that transfers glucose (Glc) from Uridine diphosphate-Glc to MGlcDAG to generate precisely that predicted LTA anchor, DGlcDAG (9). The orfB gene product situated immediately downstream of $i a g A$ in GBS shares homology to the glycosyltransferase of A. laidlawii that generates the MGlcDAG precursor. WT GBS possesses both MGlcDAG and DGlcDAG among its cell wall glycolipids, whereas the $\triangle i a g A$ mutant contained only MGlcDAG. This finding indicates that our allelic replacement of $i$ ag $A$ did not exert polar effects to impair the predicted enzymatic function of orfB. Nevertheless, with loss of the DGlcDAG synthase function encoded by iagA, cell membrane anchoring of LTA was impaired, and free LTA was shed into the media. Interestingly, a nonhomologous gene from Bacillus subtilis and $S$. aureus, ypfP, has also been shown to encode a DGlcDAG synthase (36). Disruption of ypfP in S. aureus resulted in loss of cell membrane MGlcDAG and DGlcDAG and increased release of LTA into the media (37); however, the effect of this mutation in $S$. aureus on host-cell interactions has yet to be examined.

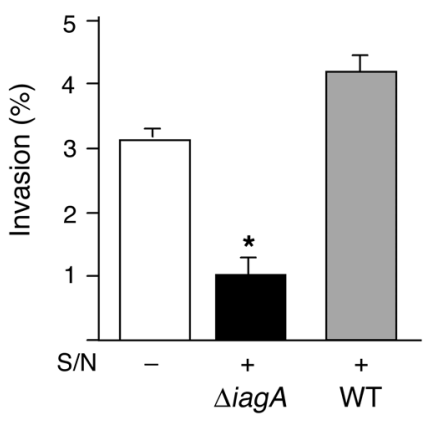

Figure 6

GBS invasion of hBMEC monolayers in the presence of supernatant from WT or $\triangle i a g A$ strains, as indicated. Supernatants $(400 \mu \mathrm{l})$ were added 1 hour prior to start of the assay. ${ }^{*} P<0.02$. 
Our discovery that cell membrane anchoring of LTA is critical for hBMEC invasion and the pathogenesis of GBS meningitis suggest that this surface polymer mediates unique host-cell interactions and provides new perspective on observations from the earlier literature. Epidemiologic investigations found that clinical isolates of GBS from infants with early- or late-onset invasive disease possess significantly higher quantities of cell-associated LTA than strains isolated from the mucosal surfaces of asymptomatically colonized infants (38). Longer LTA polymer length (30-35 GP units) appears also to be a characteristic of isolates from carriers with invasive GBS disease compared with asymptomatic carriers (10-15 GP units) (39). GBS LTA-mediated host-cell interaction involves both hydrophobic interactions with the lipid portion of the polymer as well as more specific interactions due to the GP backbone (40).

In our approach, we sought to understand whether membraneanchored LTA promoted GBS BBB invasion by facilitating direct bacterial cell interactions or indirectly, by stimulating host inflammatory responses. In many Gram-positive species, LTA (along with cell wall peptidoglycan) can stimulate mononuclear cell production of TNF- $\alpha$, IFN- $\gamma$, and inducible nitric oxide synthetase, thereby provoking septic shock, circulatory collapse, and multiple organ failure in animal models of systemic infection $(41,42)$. Underlying this proinflammatory effect is the fact that LTA is a pattern-recognition molecule for TLR2-mediated activation of the innate host immune response $(22,23)$. However, very recent studies suggest that the LTA of GBS is itself a relatively weak stimulus that does not contribute significantly to GBS cell wall-mediated macrophage activation (43). In the present study, our experiments showed that the attenuated phenotype of the $\Delta i a g A$ mutant was preserved in TLR2 ${ }^{-/}$animals and that WT and $\triangle i a g A$ mutants were equivalent in their ability to stimulate TLR2-dependent NF- $\mathrm{KB}$ activation in endothelial cells and IL-8 release from hMBECs. Thus, our data strongly suggest that the attenuated phenotype of the $\triangle i a g A$ mutant is not dependent on TLR2.

Association of GBS with human epithelial cells is inhibited by addition of soluble LTA but not by the group B carbohydrate antigen or serotype III capsular polysaccharide (40). A recent report showed that free LTA specifically blocks intracellular invasion of Hep2 pharyngeal epithelial cells by $S$. pyogenes without affecting overall cellular adherence (44). In vivo, topical administration of LTA has also been shown to be effective in blocking vaginal colonization of pregnant mice by GBS (45). Our findings in invasion assays performed in the presence of $\triangle i a g A$ supernatants containing high levels of released LTA appear to support these earlier observations. We speculate that loss of the DGlcDAG cell membrane anchor for LTA not only removes a potential "invasin" from the GBS surface but that the resultant shed LTA may also act as a competitive inhibitor for hBMEC uptake mechanisms. Our discovery of the novel requirement of LTA anchoring for GBS BBB endothelial invasion suggests that therapies targeted at neutralizing this surface molecule could be beneficial in preventing development of meningitis in infants with systemic GBS infection.

\section{Methods}

Bacterial strains and culture conditions. GBS WT strains used were COH1, a highly encapsulated serotype III clinical isolate (46) with proven virulence in animal models of sepsis and meningitis $(14,47)$, and A909, a serotype Ia strain. $\mathrm{COH} 1-13$ is an isogenic, capsule-deficient variant of $\mathrm{COH} 1$ generated upon disruption of the $\operatorname{cps} \mathrm{E}$ capsule biosynthesis gene by $\operatorname{Tn} 916 \Delta \mathrm{E}$ mutagenesis (48). GBSs were grown in THB (Difco; BD Diagnostics) as standing cultures at $37^{\circ} \mathrm{C}$. For collection of bacterial supernatants, strains were grown in hBMEC medium at $37^{\circ} \mathrm{C}$ with $5 \% \mathrm{CO}_{2}$. For antibiotic selection, $5 \mu \mathrm{g} / \mathrm{ml}$ of erythromycin (Em) or $2 \mu \mathrm{g} / \mathrm{ml}$ of chloramphenicol $(\mathrm{Cm})$ were used. E. coli strains were grown in Luria-Bertani broth with aeration; antibiotic selection employed $500 \mu \mathrm{g} / \mathrm{ml}$ of Em or $10 \mu \mathrm{g} / \mathrm{ml}$ of Cm was used for antibiotic selection.

Construction of GBS mutant libraries. Random transposon mutagenesis of GBS COH1 was accomplished by introduction of Tn917 on conditional vector $\mathrm{PTV}_{1} \mathrm{OK}$ via electroporation as described previously (49). Transformants were grown at the permissive temperature $\left(30^{\circ} \mathrm{C}\right)$ under Em selection, and chromosomal $\mathrm{Tn} 917$ transposition events were identified in colonies exhibiting Em resistance at the nonpermissive temperature $\left(37^{\circ} \mathrm{C}\right)$. Analysis of a random sampling of these isolates by Southern blot analysis using a digoxigenin-labeled (Roche Diagnostic Corp.) Tn917 probe demonstrated a random chromosomal distribution of Tn917 insertion and a single integrated copy of Tn917 in more than $80 \%$ of the mutants (data not shown). We accomplished random plasmid integrational mutagenesis of GBS strain COH1-13 by first constructing a GBS genomic library in the conditional vector pVE6007 (50). Total GBS chromosomal DNA was isolated from strain $\mathrm{COH} 1$ as described previously (49). A partial Sau3A digest was performed, and fragments of approximately 300-500 bp in size were isolated on a sucrose gradient and cloned into the BamH1-digested pVE6007 using standard techniques. This library was introduced by electroporation into COH1-13 to identify hBMEC invasion determinants that are independent of encapsulation. Plasmid integrational mutants, the product of homologous recombination directed by the cloned intragenic fragments, were selected on $\mathrm{Cm}$ at the nonpermissive temperature $\left(37^{\circ} \mathrm{C}\right)$.

Human BBB model. A well-characterized hBMEC line, immortalized by transfection with the SV40 large T antigen (5), was generously provided by Kwang Sik Kim (Johns Hopkins University, Baltimore, Maryland, USA). These hBMECs maintain the morphologic and functional characteristics of primary brain endothelial cells and have been used to successfully model human BBB interactions with host cells and infectious microorganisms (14). Cell maintenance and assays for GBS hBMEC adherence, invasion, and intracellular survival were performed as described previously $(6,51)$. For all experiments, hBMECs were used at passage 8-14. In one experiment, hBMECs were pretreated with $10 \mathrm{ng} / \mu \mathrm{l}$ of cytokine TNF- $\alpha$, IL-6, or IL-8 for 1 hour prior to the assay. Parallel invasion experiments were performed with A549 lung epithelial cells (52) and JEG-3 chorion epithelial cells obtained from ATCC. Initial screening of GBS mutant libraries was performed using a modified hBMEC invasion assay in a 96-well microtiter plate (53). Mutants exhibiting less than $50 \%$ of the invasion levels observed in the WT strain in the screening assay were selected for further analysis.

Identification of mutant genes. The site of Tn917 or plasmid insertion was mapped in hypo-invasive mutants identified in the screening hBMEC invasion assay. For Tn917, a single-primer PCR method (54) was adapted using an outwardly directed Tn917 primer, 5'-CTAAACACTTAAGAGAATTG-3', to generate PCR amplicons of flanking chromosomal DNA and a nested primer, 5'-CGCCTTGAAACATTGGTT-3', for sequencing. To identify chromosomal insertions in the pVE6007 integrational library, the mutant in question was passed in THB plus $\mathrm{Cm}$ at $30^{\circ} \mathrm{C}$ to allow for plasmid excision and extrachromosomal replication. The plasmid was purified and the GBS DNA insert sequenced directly using plasmid-specific primers. The BLAST algorithm (55) was used to compare identified sequences with the sequences of the 2 published GBS genomes $(7,8)$ and all entries in the GenBank database.

Targeted mutagenesis and complementation vector construction. PCR was used to generate the in-frame substitution of the iagA gene with the chloramphenicol acetyltransferase (cat) gene. Briefly, approximately 500 bp of sequence immediately upstream of iagA was amplified with the primers iagAupF, 
5'-CAGAACTAGTGCATGCGAGAATTTGGCCAACCATCC-3', and iagAstartR, 5'-GGTGGTATACCAGTGATTTTTTTCTCGTAAGGTCTTATTATATCTCATTTTAC-3' ${ }^{\prime}$, and approximately 500 bp immediately downstream of iagA amplified with the primers iagAendF, $5^{\prime}$-TACTGCGATGAGTGGCAGGGGGGGCGTAAGATGCGTATAGGTCTATTTACAGA-3', and iagAdownR, 5'-CAGAGGATCCCACTTAGGTAGGTACGCATG-3'. The iagAstartR and iagAendF primers were constructed with 25-bp $5^{\prime}$ extensions corresponding to the $5^{\prime}$ and $3^{\prime}$ ends of the cat gene, respectively. The upstream and downstream PCR products were then combined with a 650bp amplicon of the complete cat gene (from pACYC184) as templates in a second round of PCR using primers iagAupF and iagAupR. The resultant PCR amplicon, containing an in-frame substitution of $i a g A$ with cat, was subcloned into temperature-sensitive vector $\mathrm{pHY} 304$, and allelic exchange mutagenesis of $\mathrm{COH} 1$ was performed as described previously (56) to generate the stable mutant COH1 $\triangle i a g A$. Precise in-frame, allelic replacement of iagA with cat was confirmed by PCR on COH1 1 iagA chromosomal DNA. For complementation studies, iagA plus flanking DNA was PCR amplified from the $\mathrm{COH} 1$ chromosome using iagAupF and iagAupR and cloned into shuttle expression vector pDCerm (57), yielding plasmid pDCiagA for complementation analysis. Disruption of the iagA gene was also performed in the capsule-deficient derivative of COH1 and a type Ia GBS strain A909.

Animal infection studies. All animal experiements were approved by the Committee on the Use and Care of Animals, UCSD, and performed using accepted veterinary standards. Experiments assessing GBS survival in human whole blood were performed using bacterial counts of $10^{4}-10^{6}$ as described previously (58). A murine model of hematogenous GBS meningitis has been described previously (14). Briefly, outbred 6-week-old male CD-1, C57BL/6 (Charles River Laboratories), and C57BL/6 TLR2 ${ }^{-/-}$were injected via tail vein with $2 \times 10^{8}$ to $5 \times 10^{8} \mathrm{CFU}$ of WT GBS COH 1 or the $\triangle$ iagA mutant ( $n=5-10$ per group). At 6-20 hours after injection, blood was collected from the tail vein and plated on THB agar so that the level of bacteremia could be determined. Blood, spleen, and brains were collected aseptically from mice at the time they succumbed to infection or upon euthanasia at the experimental endpoint. Bacterial counts in blood and spleen or brain homogenates were determined by plating serial 10-fold dilutions on THB agar. Brain bacterial counts were corrected for blood contamination as described previously (14).

Antimicrobial assay. Assays to determine sensitivity to the murine antimicrobial peptide CRAMP were performed as described previously (17). For analysis of antimicrobial killing kinetics, approximately $2 \times 10^{5} \mathrm{CFU}$ of each strain were exposed to $16 \mu \mathrm{M}$ CRAMP and incubated at $37^{\circ} \mathrm{C}$. Dilutions were plated at time points from 0 to 60 minutes for determination of the CFU of surviving bacteria.

Glycolipid and LTA analysis. Polar lipids from GBS were separated by 1dimensional thin-layer chromatography in chloroform/methanol/water (70:30:4, vol/vol/vol). To visualize glycolipids, we sprayed the samples with $\alpha$-naphthol (3.2\%) in methanol- $\mathrm{H}_{2} \mathrm{SO}_{4}-\mathrm{H}_{2} \mathrm{O}(25: 3: 2, \mathrm{vol} / \mathrm{vol} / \mathrm{vol})$ and heated the plate at $110^{\circ} \mathrm{C}$ for $5-10$ minutes. An LTA ELISA was developed using a $\mathrm{mAb}$ against $\mathrm{LTA}, \mathrm{IgG}_{3}$ clone 55 (Hycult Biotechnology), originally derived from the splenocytes of mice challenged with Streptococcus sobrinus (20) and recognizing the common polyglycerol backbone of LTA that is shared by a large number of streptococcal and staphylococcal isolates (59). Briefly, 100 $\mu$ l of GBS supernatants from culture in THB or whole GBS cells washed several times and resuspended in THB were added to 96-well polystyrene plates (Immulon 4, Dynatech Laboratories) and incubated overnight at $4^{\circ} \mathrm{C}$. A standard curve was generated using purified LTA from S. aureus (SigmaAldrich) $(0-100 \mathrm{ng} / \mathrm{ml}$ in THB). For detection, anti-LTA antibody was used at 1:125 for 1 hour followed by a 1:500 dilution of goat anti-mouse IgGHRP conjugate (Sigma-Aldrich) for 90 minutes at room temperature. The LTA concentration was calculated against the standard curve.

IL-8 ELISA. hBMECs were exposed to WT or the $\triangle i a g A$ mutant strain for 4 or 8 hours, after which supernatants were collected and analyzed for secreted IL-8 protein using ELISA as described previously (14).

TLR2 transfection and signaling. The immortalized human dermal endothelial cells, HMECs (a generous gift of F.J. Candal of the Centers for Disease Control, Atlanta, Georgia, USA), were cultured in MCDB-131 medium with $10 \%$ heat-inactivated FBS, $2 \mathrm{mM}$ glutamine, and $100 \mu \mathrm{g} / \mathrm{ml}$ penicillin and streptomycin plated at a concentration of 50,000 cells/well in 24-well plates. It has been shown previously that HMECs do not express TLR 2 or TLR4 and do not respond to TLR2 or TLR4 ligands by NF- $\mathrm{KB}$ activation (60). Cells were transfected using FuGENE 6 Transfection Reagent (Boehringer Mannheim) according to the manufacturer's instructions in RPMI with $10 \%$ serum. Reporter genes pCMV-galactosidase $(0.1 \mu \mathrm{g})$ and ELAM-NF-KB-luciferase $(0.5 \mu \mathrm{g})$, and pcDNA3 empty vector, TLR4 or TLR2 cDNA ( $0.5 \mu \mathrm{g}$ each), were used. After a 24 -hour transfection, cells were stimulated for 5 hours with WT or the $\triangle i a g A$ mutant GBS or TLR2 ligand phenol-soluble modulin (100 ng/ml; ref. 61) as positive control. Cells were then lysed, and luciferase activity was measured with a Promega kit E4550 and a luminometer. Galactosidase activity was determined by the calorimetric method to normalize transfection efficiency as described previously (62). Data shown represent the mean of 2 independent experiments.

Statistical analysis. Differences in bacterial cfu or LTA were evaluated by a 2-tailed Student's $t$ test.

\section{Acknowledgments}

The authors are grateful to Monique Stins and Kwan Sik Kim for providing hBMECs and Amanda Lewis for HPLC analysis. Histopathology studies were performed at the Histopathology Core Facility at UCSD, Director Nissi Varki. This work was supported by a Burroughs Wellcome Fund Career Award (to K.S. Doran), an American Heart Association Established Investigator Award (to V. Nizet), the Edward J. Mallinckrodt, Jr., Foundation, the NIH (HD37224), and the United Cerebral Palsy Research Foundation.

Received for publication November 5, 2004, and accepted in revised form June 14, 2005.

Address correspondence to: Kelly S. Doran, University of California, San Diego, School of Medicine, Center for Molecular Medicine East, Room 1059, 9500 Gilman Drive, Mail Code 0687, La Jolla, California 92093, USA. Phone: (858) 822-4260; Fax: (858) 534-5611; E-mail: kdoran@ucsd.edu.
1. Leib, S.L., and Tauber, M.G. 1999. Pathogenesis of bacterial meningitis [review]. Infect. Dis. Clin. North Am. 13:527-548, v-vi.

2. Betz, A.L. 1985. Epithelial properties of brain capillary endothelium. Fed. Proc. 44:2614-2615.

3. Baker, C.J., and Edwards, M.S. 1995. Group B streptococcal infections. In Infectious diseases of the fetus and newborn infant. J. Remington and J.O. Klein, editors. W.B. Saunders. Philadelphia, Pennsylvania, USA. $980-1054$.
4. Ferrieri, P., Burke, B., and Nelson, J. 1980. Production of bacteremia and meningitis in infant rats with group B streptococcal serotypes. Infect. Immun. 27:1023-1032.

5. Stins, M.F., Prasadarao, N.V., Zhou, J., Arditi, M., and Kim, K.S. 1997. Bovine brain microvascular endothelial cells transfected with SV40-large T antigen: development of an immortalized cell line to study pathophysiology of CNS disease. In Vitro Cell. Dev. Biol. Anim. 33:243-247.
6. Nizet, V., et al. 1997. Invasion of brain microvascular endothelial cells by group B streptococci. Infect. Immun. 65:5074-5081.

7. Glaser, P., et al. 2002. Genome sequence of Streptococcus agalactiae, a pathogen causing invasive neonatal disease. Mol. Microbiol. 45:1499-1513.

8. Tettelin, H., et al. 2002. Complete genome sequence and comparative genomic analysis of an emerging human pathogen, serotype V Streptococcus agalactiae. Proc. Natl. Acad. Sci. U. S. A. 99:12391-12396. 
9. Lindblom, G., Brentel, I., Sjolund, M., Wikander, G., and Wieslander, A. 1986. Phase equilibria of membrane lipids from Acholeplasma laidlawii: importance of a single lipid forming nonlamellar phases. Biochemistry. 25:7502-7510.

10. Grebe, T., Paik, J., and Hakenbeck, R. 1997. A novel resistance mechanism against beta-lactams in Streptococcus pneumoniae involves $\mathrm{C}$ poA, a putative glycosyltransferase. J. Bacteriol. 179:3342-3349.

11. Winram, S.B., Jonas, M., Chi, E., and Rubens, C.E. 1998. Characterization of group B streptococcal invasion of human chorion and amnion epithelial cells in vitro. Infect. Immun. 66:4932-4941.

12. Nizet, V., et al. 1996. Group B streptococcal betahemolysin expression is associated with injury of lung epithelial cells. Infect. Immun. 64:3818-3826.

13. Doran, K.S., and Nizet, V. 2004. Molecular pathogenesis of neonatal group B streptococcal infection: no longer in its infancy [review]. Mol. Microbiol. 54:23-31.

14. Doran, K.S., Liu, G.Y., and Nizet, V. 2003. Group B streptococcal $\beta$-hemolysin/cytolysin activates neutrophil signaling pathways in brain endothelium and contributes to development of meningitis. J. Clin. Invest. 112:736-744. doi:10.1172/ JCI200317335.

15. Lewis, A.L., Nizet, V., and Varki, A. 2004. Discovery and characterization of sialic acid O-acetylation in group B Streptococcus. Proc. Natl. Acad. Sci. U. S. A. 101:11123-11128.

16. Weisner, A.M., et al. 2004. Characterization of group B streptococci recovered from infants with invasive disease in England and Wales. Clin. Infect. Dis. 38:1203-1208.

17. Nizet, V., et al. 2001. Innate antimicrobial peptide protects the skin from invasive bacterial infection. Nature. 414:454-457.

18. Iordanescu, S., and Surdeanu, M. 1976. Two restriction and modification systems in Staphylococcus aureus NCTC8325. J. Gen. Microbiol. 96:277-281.

19. Neuhaus, F.C., and Baddiley, J. 2003. A continuum of anionic charge: structures and functions of D-alanyl-teichoic acids in gram-positive bacteria [review]. Microbiol. Mol. Biol. Rev. 67:686-723.

20. Hogg, S.D., Whiley, R.A., and De Soet, J.J. 1997. Occurrence of lipoteichoic acid in oral streptococci. Int. J. Syst. Bacteriol. 47:62-66.

21. Schwandner, R., Dziarski, R., Wesche, H., Rothe, M., and Kirschning, C.J. 1999. Peptidoglycan- and lipoteichoic acid-induced cell activation is mediated by toll-like receptor 2. J. Biol. Chem. 274:17406-17409.

22. Takeuchi, O., et al. 1999. Differential roles of TLR2 and TLR4 in recognition of gram-negative and gram-positive bacterial cell wall components. Immunity. 11:443-451.

23. Mancuso, G., et al. 2004. Dual role of TLR2 and myeloid differentiation factor 88 in a mouse model of invasive group B streptococcal disease. J. Immunol. 172:6324-6329.

24. Andres, O., Obojes, K., Kim, K.S., ter Meulen, V., and Schneider-Schaulies, J. 2003. CD46- and CD150independent endothelial cell infection with wildtype measles viruses. J. Gen. Virol. 84:1189-1197.

25. Ring, A., Weiser, J.N., and Tuomanen, E.I. 1998. Pneumococcal trafficking across the blood-brain barrier. Molecular analysis of a novel bidirectional pathway. J. Clin. Invest. 102:347-360.

26. Huang, S.H., Stins, M.F., and Kim, K.S. 2000. Bacterial penetration across the blood-brain barrier during the development of neonatal meningitis. Microbes Infect. 2:1237-1244.

27. Hertzig, T., et al. 2003. Antibodies present in normal human serum inhibit invasion of human brain microvascular endothelial cells by Listeria monocytogenes. Infect. Immun. 71:95-100.

28. Cieslewicz, M.J., Kasper, D.L., Wang, Y., and Wes- sels, M.R. 2001. Functional analysis in type Ia group B Streptococcus of a cluster of genes involved in extracellular polysaccharide production by diverse species of streptococci. J. Biol. Chem. 276:139-146.

29. Chaffin, D.O., Beres, S.B., Yim, H.H., and Rubens, C.E. 2000. The serotype of type Ia and III group B streptococci is determined by the polymerase gene within the polycistronic capsule operon. J. Bacteriol. 182:4466-4477.

30. Rubens, C.E., Wessels, M.R., Heggen, L.M., and Kasper, D.L. 1987. Transposon mutagenesis of type III group B Streptococcus: correlation of capsule expression with virulence. Proc. Natl. Acad. Sci.U. S. A. 84:7208-7212.

31. Marques, M.B., Kasper, D.L., Pangburn, M.K., and Wessels, M.R. 1992. Prevention of C3 deposition by capsular polysaccharide is a virulence mechanism of type III group B streptococci. Infect. Immun. 60:3986-3993.

32. Wicken, A.J., and Knox, K.W. 1975. Lipoteichoic acids: a new class of bacterial antigen [review]. Science. 187:1161-1167.

33. Fischer, W. 1988. Physiology of lipoteichoic acids in bacteria. Adv. Microb. Physiol. 29:233-302.

34. Goldschmidt, J.C., Jr., and Panos, C. 1984. Teichoic acids of Streptococcus agalactiae: chemistry, cytotoxicity, and effect on bacterial adherence to human cells in tissue culture. Infect. Immun. 43:670-677.

35. Mattingly, S.J., and Johnston, B.P. 1987. Comparative analysis of the localization of lipoteichoic acid in Streptococcus agalactiae and Streptococcus pyogenes. Infect. Immun. 55:2383-2386.

36. Jorasch, P., Wolter, F.P., Zahringer, U., and Heinz, E. 1998. A UDP glucosyltransferase from Bacillus subtilis successively transfers up to four glucose residues to 1,2-diacylglycerol: expression of $y p f \mathrm{P}$ in Escherichia coli and structural analysis of its reaction products. Mol. Microbiol. 29:419-430.

37. Kiriukhin, M.Y., Debabov, D.V., Shinabarger, D.L., and Neuhaus, F.C. 2001. Biosynthesis of the glycolipid anchor in lipoteichoic acid of Staphylococcus aureus RN4220: role of YpfP, the diglucosyldiacylglycerol synthase. J. Bacteriol. 183:3506-3514.

38. Nealon, T.J., and Mattingly, S.J. 1983. Association of elevated levels of cellular lipoteichoic acids of group B streptococci with human neonatal disease. Infect. Immun. 39:1243-1251.

39. Nealon, T.J., and Mattingly, S.J. 1985. Kinetic and chemical analyses of the biologic significance of lipoteichoic acids in mediating adherence of serotype III group B streptococci. Infect. Immun. 50:107-115.

40. Nealon, T.J., and Mattingly, S.J. 1984. Role of cellular lipoteichoic acids in mediating adherence of serotype III strains of group B streptococci to human embryonic, fetal, and adult epithelial cells. Infect. Immun. 43:523-530.

41. De Kimpe, S.J., Kengatharan, M., Thiemermann, C., and Vane, J.R. 1995. The cell wall components peptidoglycan and lipoteichoic acid from Staphylococcus aureus act in synergy to cause shock and multiple organ failure. Proc. Natl. Acad. Sci. U. S. A. 92:10359-10363.

42. Kengatharan, K.M., De Kimpe, S., Robson, C., Foster, S.J., and Thiemermann, C. 1998. Mechanism of gram-positive shock: identification of peptidoglycan and lipoteichoic acid moieties essential in the induction of nitric oxide synthase, shock, and multiple organ failure. J. Exp. Med. 188:305-315.

43. Henneke, P., et al. 2005. Role of lipoteichoic acid in the phagocyte response to group B streptococcus. J. Immunol. 174:6449-6455.

44. Sela, S., Marouni, M.J., Perry, R., and Barzilai, A. 2000. Effect of lipoteichoic acid on the uptake of Streptococcus pyogenes by HEp-2 cells. FEMS Micro- biol. Lett. 193:187-193.

45. Cox, F. 1982. Prevention of group B streptococcal colonization with topically applied lipoteichoic acid in a maternal-newborn mouse model. Pediatr. Res. 16:816-819.

46. Wessels, M.R., Benedi, V.-J., Kasper, D.L., Heggen, L.M., and Rubens, C.E. 1991. Type III capsule and virulence of group B streptococci. In Genetics and molecular biology of streptococci, lactococci, and enterococci. G.M. Dunny, P.P. Cleary, and L.L. McKay, editors. American Society for Microbiology. Washington, DC, USA. 219-223.

47. Puliti, M., et al. 2000. Severity of group B streptococcal arthritis is correlated with beta-hemolysin expression. J. Infect. Dis. 182:824-832.

48. Rubens, C.E., Heggen, L.M., Haft, R.F., and Wessels, M.R. 1993. Identification of cpsD, a gene essential for type III capsule expression in group B streptococci. Mol. Microbiol. 8:843-855.

49. Framson, P.E., Nittayajarn, A., Merry, J., Youngman, P., and Rubens, C.E. 1997. New genetic techniques for group B streptococci: high-efficiency transformation, maintenance of temperature-sensitive pWV01 plasmids, and mutagenesis with Tn917. Appl. Environ. Microbiol. 63:3539-3547.

50. Maguin, E., Duwat, P., Hege, T., Ehrlich, D., and Gruss, A. 1992. New thermosensitive plasmid for gram-positive bacteria. J. Bacteriol. 174:5633-5638.

51. Kim, K.S. 2001. Escherichia coli translocation at the blood-brain barrier. Infect. Immun. 69:5217-5222.

52. Doran, K.S., Chang, J.C., Benoit, V.M., Eckmann, L., and Nizet, V. 2002. Group B streptococcal betahemolysin/cytolysin promotes invasion of human lung epithelial cells and the release of interleukin-8. J. Infect. Dis. 185:196-203.

53. Nizet, V., Smith, A.L., Sullam, P.M., and Rubens, C.E. 1998. A simple microtiter plate screening assay for bacterial invasion or adherence. Methods Cell Sci. 20:107-111.

54. Karlyshev, A.V., Pallen, M.J., and Wren, B.W. 2000. Single-primer PCR procedure for rapid identification of transposon insertion sites. Biotechniques. 28:1078, 1080, 1082.

55. Altschul, S.F., and Lipman, D.J. 1990. Protein database searches for multiple alignments. Proc. Natl. Acad. Sci. U. S. A. 87:5509-5513.

56. Pritzlaff, C.A., et al. 2001. Genetic basis for the beta-haemolytic/cytolytic activity of group B Streptococcus. Mol. Microbiol. 39:236-247.

57. Jeng, A., et al. 2003. Molecular genetic analysis of a group A Streptococcus operon encoding serum opacity factor and a novel fibronectin-binding protein, SfbX. J. Bacteriol. 185:1208-1217.

58. Liu, G.Y., et al. 2004. Sword and shield: linked group B streptococcal beta-hemolysin/cytolysin and carotenoid pigment function to subvert host phagocyte defense. Proc. Natl. Acad. Sci. U. S. A. 101:14491-14496.

59. van Langevelde, P., et al. 1998. Antibiotic-induced release of lipoteichoic acid and peptidoglycan from Staphylococcus aureus: quantitative measurements and biological reactivities. Antimicrob. Agents Chemother. 42:3073-3078.

60. Faure, E., et al. 2000. Bacterial lipopolysaccharide activates NF-kappaB through toll-like receptor 4 (TLR-4) in cultured human dermal endothelial cells. Differential expression of TLR-4 and TLR- 2 in endothelial cells. J. Biol. Chem. 275:11058-11063.

61. Hajjar, A.M., et al. 2001. Cutting edge: functional interactions between toll-like receptor (TLR) 2 and TLR 1 or TLR 6 in response to phenol-soluble modulin. J. Immunol. 166:15-19.

62. Muzio, M., Ni, J., Feng, P., and Dixit, V.M. 1997. IRAK (Pelle) family member IRAK-2 and MyD88 as proximal mediators of IL-1 signaling. Science. 278:1612-1615. 